\title{
Solution-processed amorphous silicon surface passivation layers
}

\author{
Mathias Mews, ${ }^{1}$ a) Christoph Mader, ${ }^{2}$ Stephan Traut, ${ }^{2}$ Tobias Sontheimer, ${ }^{1}$ Odo Wunnicke, ${ }^{2}$ Lars Korte, ${ }^{1}$ and \\ Bernd Rech ${ }^{1}$ \\ ${ }^{1)}$ Helmholtz-Zentrum Berlin, Institute of Silicon Photovoltaics, Kekuléstraße 5, D-12489 Berlin, \\ Germany \\ ${ }^{2)}$ Evonik Industries AG, Creavis Technologies $\&$ Innovation, Paul-Baumann-Straße 1, D-45772 Marl, \\ Germany
}

(Dated: 26 September 2014)

Amorphous silicon thin films, fabricated by thermal conversion of neopentasilane, were used to passivate crystalline silicon surfaces. The conversion is investigated using X-ray and constant-final-state-yield photoelectron spectroscopy, and minority charge carrier lifetime spectroscopy. Liquid processed amorphous silicon exhibits high Urbach energies from 90 to $120 \mathrm{meV}$ and $200 \mathrm{meV}$ lower optical band gaps than material prepared by plasma enhanced chemical vapor deposition. Applying a hydrogen plasma treatment, a minority charge carrier lifetime of $1.37 \mathrm{~ms}$ at an injection level of $10^{15} / \mathrm{cm}^{3}$ enabling an implied open circuit voltage of $724 \mathrm{mV}$ was achieved, demonstrating excellent silicon surface passivation.

Today the most widely used semiconductor material is silicon, applied as crystalline wafers and amorphous, microcrystalline, or polycrystalline layers for photovoltaic and electronic applications. In many cases the material preparation involves complex vacuum processes and laterally structured devices have to be realized using costly processes like photolithography. Printing or drop-casting a liquid silicon precursor and converting it afterwards to amorphous or crystalline layers would offer great potential for simplified fabrication of silicon based semiconductor devices.

Solution processed intrinsic amorphous silicon (a-Si:H) could find applications as buffer layer in high-efficiency amorphous-crystalline silicon hetero-junction (a-Si:H/cSi-SHJ) solar cells ${ }^{1}$, as capping layer for thin $\mathrm{AlO}_{x}$ rearside passivation layers of p-type $\mathrm{c}$-Si solar cells ${ }^{2}$, or as surface passivation in light emitting devices ${ }^{3}$. Other potential applications for solution processed a-Si:H layers are electron and hole collector layers in amorphouscrystalline silicon bipolar transistors ${ }^{4}$, and a-Si:H/c-Si solar cells ${ }^{5}$ in conventional as well as in back-contact backjunction configuration.

First experiments with liquid polysilane precursors such as cyclopentasilane ${ }^{6}$, neopentasilane ${ }^{7}$ or trisilane $^{8}$ were conducted to manufacture a variety of structures and devices. Amorphous silicon (a-Si:H) thin films were applied in solar cells ${ }^{9,10}$, light-emitting devices ${ }^{11}$ and thin-film transistors $^{6}$. Furthermore crystalline silicon nanowires were used as anode material in lithium batteries ${ }^{12}$.

This contribution aims at investigating the conversion from neopentasilane to amorphous silicon and evaluating the potential of solution processed a-Si:H layers for surface passivation of crystalline silicon. First the conversion from the polysilane precursor to a-Si:H is investigated using X-ray photoelectron spectroscopy (XPS). Second the electronic properties of spin-coated amorphous silicon are compared to state of the art amorphous silicon

\footnotetext{
a) mathias.mews@helmholtz-berlin.de
}

deposited using plasma-enhanced chemical vapor deposition (PECVD). Finally the minority carrier passivation achieved with spin-coated a-Si:H and polysilane layers is discussed.

$165 \mu \mathrm{m}$ thick, (100) oriented and damage etched n-type $\mathrm{Cz}$ silicon wafers with a resistivity of $3-5 \Omega \mathrm{cm}$ were used as substrates for passivation experiments. To exclude any influence of damage to the wafer backside during processing and to provide rear surface passivation, the wafer backsides were coated with $10 \mathrm{~nm} \mathrm{AlO}_{x}$ and $90 \mathrm{~nm}$ $\mathrm{SiN}_{x}{ }^{13}$. P-type (100) oriented chemically-mechanically polished $500 \mu \mathrm{m}$ thick $\mathrm{Cz}$ silicon wafers were used as substrates for photoeletron spectroscopy (PES).

The liquid silicon layers were prepared following an approach, presented in an earlier publication ${ }^{7}$. Briefly neopentasilane $\left(\mathrm{Si}_{5} \mathrm{H}_{12}\right)^{14}$ was oligomerized by thermal treatment and dissolved in toluene and cyclooctane, yielding a $30 \mathrm{wt} \%$ solution. Afterwards 50 to $90 \mathrm{~nm}$ thin films were prepared using spin-coating and subsequent annealing on a hot plate at temperatures between 250 and $600^{\circ} \mathrm{C}$ for $10 \mathrm{~s}$ to $30 \mathrm{~s}$. All process steps were carried out in nitrogen atmosphere to prevent oxidation of the neopentasilane.

To further decrease the defect density in the a-Si:H layers and at the a-Si:H/c-Si interface hydrogen plasma post-deposition treatments were applied ${ }^{15}$ in a parallel plate plasma enhanced chemical vapor deposition reactor (PECVD). The process pressure was $0.4 \mathrm{mbar}$, the excitation frequency $60 \mathrm{MHz}$, the substrate temperature $195^{\circ} \mathrm{C}$, the distance between electrode and substrate $16 \mathrm{~mm}$ and the plasma power density $115 \mathrm{~mW} / \mathrm{cm}^{2}$.

A Sinton Consulting WCT-100 photoconductance decay measurement setup was used for lifetime measurements ${ }^{16}$. PES was executed using either $\mathrm{Mg}-\mathrm{K}_{\alpha}$ excitation for core level spectroscopy or variable photon energy in the near-UV range, from 3.7 to $7.3 \mathrm{eV}$, to conduct PES in the constant-final-state-yield mode $(\mathrm{CFSYS})^{17}$. A model density of states $^{18}$ was fitted to the data to obtain the valence band position and Urbach tail slope of the a-Si:H layers. The samples were transported in nitrogen atmosphere from the preparation 


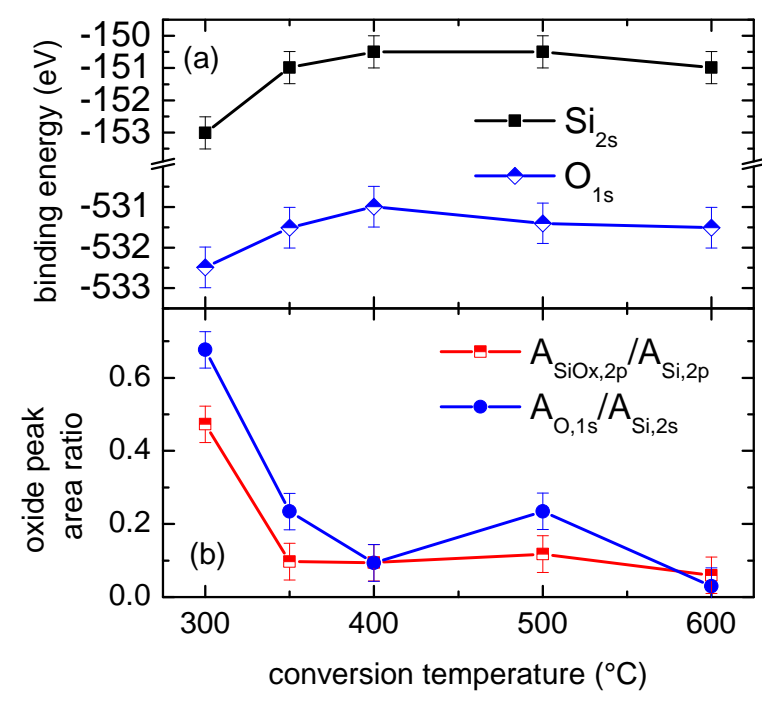

FIG. 1. (a) Dependence of the Si 2s and O 1s binding energy on the conversion temperature. (b) Conversion temperature dependence of the ratio between the silicon oxide and the silicon peak area, as determined from the $\mathrm{O}$ 1s and $\mathrm{Si} 2 \mathrm{~s}$ peaks and from the components of the $\mathrm{Si} 2 \mathrm{p}$ peak. Samples were transported in nitrogen atmosphere and were Ar sputtercleaned prior to measurements.

setup to the PES setup, to limit the influence of oxidation on the PES measurements. Since sample transfer from the transport equipment to the PES setup is not possible without breaching the inert atmosphere, the samples were exposed to air for a short time $(<5 \mathrm{~min})$. Afterwards the samples were sputtered in an argon plasma to remove oxidized regions. The sputtering conditions were optimized to remove only the oxidized surface layer. Core-level spectroscopy using XPS was conducted after sputtering, while CFSYS measurements of the valence band density of states were carried out before sputtering, as the sputtering process alters the valence band properties significantly. Note, that at the low excitation energies used for CFSYS, the information depth is about $5-10 \mathrm{~nm}$ and therefore the influence of the surface on the spectrum is small.

To resolve the chemical conversion from neopentasilane to amorphous silicon its dependence on the treatment temperature was investigated using XPS measurements of $50 \mathrm{~nm}$ thick films converted at temperatures ranging from 300 to $600^{\circ} \mathrm{C}$. The dependence of the Si $2 \mathrm{~s}$ and $\mathrm{O} 1 \mathrm{~s}$ core level binding energies on the conversion temperature is shown in Fig. 1a. For temperatures below $400^{\circ} \mathrm{C}$ the binding energy of the peaks increases, due to a higher oxygen fraction in these samples ${ }^{19}$.

The Si 2p core levels dependence on conversion temperature will be discussed in the following (see Fig. 1b). Note that the spin-orbit splitting of the Si $2 p$ peak is not discernible within the resolution of the used measurement system. The four oxidation states of the silicon $2 \mathrm{p}^{0}$ are distributed between $100 \mathrm{eV}$ and $105 \mathrm{eV}^{20}$. XPS measurements on polysilane molecule films place their Si $2 p$ binding energy at about $102.5 \mathrm{eV}^{21}$. Therefore a core-level PES signal from polysilane would be difficult to distinguish from the different $\mathrm{SiO}_{x}$ phases also present in the samples. Following an approach proposed by Hansch et $a l .{ }^{22}$ to distinguish contributions of $\mathrm{SiO}_{x}$ from those of $\mathrm{SiN}_{x}$ to the Si 2p core level spectrum, the ratio of the $\mathrm{O}$ 1s to the Si 2 s core level intensity was compared to the ratio of the higher energy contributions of the Si $2 p$ signal to the Si $2 \mathrm{p}^{0}$ signal. This data is shown in Fig. 1b. The ratio of the $\mathrm{O} 1 \mathrm{~s}$ to the $\mathrm{Si} 2 \mathrm{~s}$ signal is higher than the ratio of $\mathrm{Si} 2 \mathrm{p}^{+1,+2,+3,+4}$ to the $\mathrm{Si} 2 \mathrm{p}^{0}$ component. Therefore all contributions to the $\mathrm{Si} 2 \mathrm{p}$ signal between 100 and $105 \mathrm{eV}$ can be attributed to $\mathrm{SiO}_{x}$. Accordingly, no polysilane is detected, i.e. the whole polysilane component of these layers is oxidized during sample transfer. Moreover the total intensity of oxidic components after sputtering increases with decreasing conversion temperature from $400^{\circ} \mathrm{C}$ downwards. Therefore the conversion from polysilane to amorphous silicon occurs between 300 to $400^{\circ} \mathrm{C}$ and at $400^{\circ} \mathrm{C}$ the whole precursor is fully converted. Note that the sample, annealed at $500^{\circ} \mathrm{C}$, still shows a small $\mathrm{O} 1 \mathrm{~s}$ signal i.e. some residual oxygen. As this oxygen is not reflected in the sample's Si 2p signal, it must be due to to a residual surface contamination related to the shorter sputtering time for this sample.

To sum up the preceding paragraphs, it was found that the chemical conversion from neopentasilane to amorphous silicon is possible for conversion temperatures of $400^{\circ} \mathrm{C}$ and above. To obtain additional information about the electronic structure of the valence band of these layers, CFSYS measurements were conducted. A PECVD reference layer was transferred to the PES setup through atmosphere, in order to obtain similar surface properties as for the liquid processed a-Si:H.

UV-CFSYS can be used to obtain the valence band position of a-Si:H. The valence band positions of the PECVD deposited reference layer and three liquid processed layers are depicted in Fig. 2a. The valence band of the thermally converted layers is closer to the Fermi level than for the PECVD layer and moves even closer with increasing conversion temperature. This is consistent with a lower hydrogen content in these layers and a further decrease of it, with increasing conversion temperature ${ }^{23}$. Moreover a low hydrogen content is supposedly detrimental to the electronic quality of a-Si:H.

The second parameter extracted from CFSYS data is the valence tail Urbach energy. It is a measure of electronic and structural disorder in a-Si:H, since the valence band tail consists of Si-Si-binding states with bond lengths and angles deviating from the eqilibrium ${ }^{24}$. In general a low Urbach energy of about $60 \mathrm{meV}$ is considered favorable for application of a-Si:H in electronic devices $^{23}$. The PECVD reference layer exhibits an Urbach energy of about $55 \mathrm{meV}$ (Fig. 2b). The liquid processed layers converted at $400^{\circ} \mathrm{C}$ exhibit an Urbach en- 


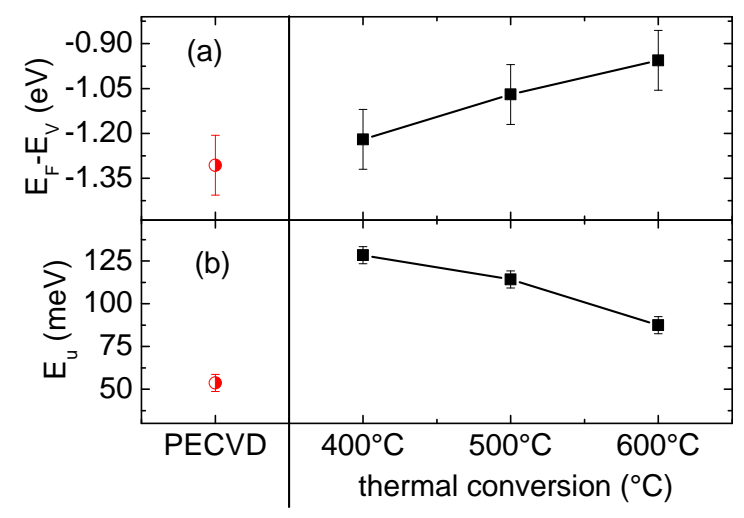

FIG. 2. Valence band position $\mathrm{E}_{\mathrm{F}}-\mathrm{E}_{\mathrm{V}}$ (a) and valence band tail Urbach energy $E_{u}$ (b) for different amorphous silicon layers. The values for a PECVD deposited $10 \mathrm{~nm}$ thin layer are displayed on the left-hand side. Values for $50 \mathrm{~nm}$ thick amorphous silicon layers, prepared by thermal conversion of neopentasilane at three different temperatures for $30 \mathrm{~s}$, are displayed on the right-hand side.

ergy of about $125 \mathrm{meV}$, which decreases with increasing conversion temperature to about $115 \mathrm{meV}$ for conversion at $500^{\circ} \mathrm{C}$ and to about $90 \mathrm{meV}$ for $600^{\circ} \mathrm{C}$. This result indicates poor structural quality and may be disadvantageous for application as electron contact, hole contact, or as buffer layer in solar cells. In contrast the structural bulk quality is less important for surface passivation layers.

Passivation of crystalline silicon with a-Si:H relies on two mechanisms: Formation of an atomically sharp a-Si:H/cSi-interface consisting mainly of Si-Si bonds and saturation of the remaining dangling bonds with hydrogen provided during and after the a-Si:H deposition ${ }^{23}$. The neopentasilane precursor transforms into a-Si:H by $\mathrm{Si}-\mathrm{H}$ bond breaking and formation of Si-Si-bonds. Therefore the hydrogen concentration in the precursor decreases during thermal conversion and is about $10 \%$ at $400^{\circ} \mathrm{C}^{25}$. Thus the conversion temperature has a strong impact on interface passivation, as a higher temperature increases the Si-Si-bond fraction, but at the same time decreases the amount of hydrogen in the layer which is needed to saturate dangling bonds at the amorphous/crystalline interface.

To investigate the influence of the treatment temperature a series of samples with different treatment temperatures and times was prepared. The minority charge carrier lifetimes at an injection level of $10^{15} \mathrm{~cm}^{-3}\left(\tau_{15}\right)$ obtained for different treatment conditions with $60 \mathrm{~nm}$ thick liquid processed layers are depicted in Fig. 3a. Note that these samples have an $\mathrm{AlO}_{x} / \mathrm{SiN}_{x}$ passivation stack on the rear side. $\mathrm{AlO}_{x}$ passivation is known to improve by thermal annealing ${ }^{26}$. Nonetheless any influence of this effect can be discounted in the present experiment, as the minority carrier lifetime enabled by the unannealed $\mathrm{AlO}_{x} / \mathrm{SiN}_{x}$ stack on the used substrates is already in the

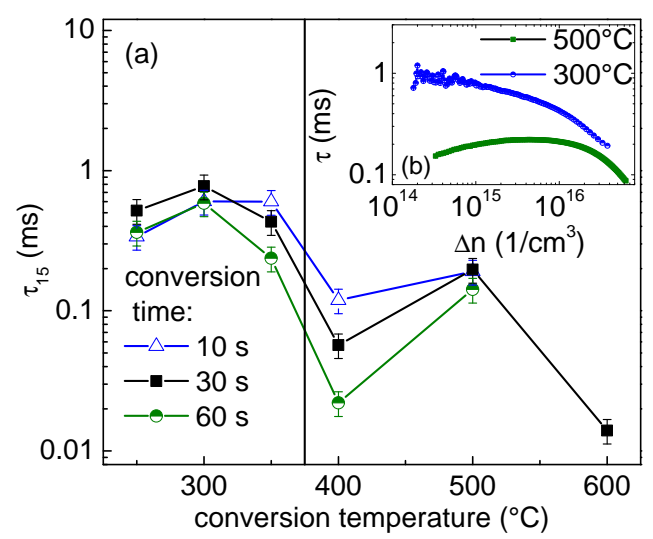

FIG. 3. (a) Dependence of the minority charge carrier lifetime at an injection level of $10^{15} \mathrm{~cm}^{-3}\left(\tau_{15}\right)$ on the conversion temperature. The passivation layers are $60 \mathrm{~nm}$ thick. The vertical line marks the approximate position of the polysilane-toamorphous silicon transition. (b) Dependence of the minority charge carrier lifetime $\tau$ on the injection carrier density $\Delta \mathrm{n}$, for two samples treated at 300 and $500^{\circ} \mathrm{C}$, respectively.

range of $2.4 \mathrm{~ms}$, thus well above any minority charge carrier lifetime measured in this series. Furthermore it was demonstrated that these $\mathrm{AlO}_{x} / \mathrm{SiN}_{x}$ stacks are stable for firing steps with process temperatures of up to $910^{\circ} \mathrm{C}$ and still allow for minority carrier lifetimes of about $5 \mathrm{~ms}$ after firing ${ }^{13}$. Therefore the sample surface passivated by neopentasilane, amorphous silicon, or any intermediate state is limiting and determines the measured effective minority charge carrier lifetime.

Three temperature series with different thermal treatment times have been prepared and all show similar tendencies. The highest $\tau_{15}$ is reached with annealing at about $300^{\circ} \mathrm{C}$. Unfortunately the passivation obtained by application of these layers degrades after a few minutes in air to a few microseconds and cannot be reestablished with thermal annealing. Furthermore the dependence of $\tau$ on the minority carrier density for these samples shows, that passivation is due to a field effect, i.e. a fixed charge close to the interface. Minority carrier lifetime curves for samples treated at 300 and $500^{\circ} \mathrm{C}$ are shown in Fig. 3b. The shape of the curves ${ }^{27}$ shows that the main passivation mechanism at 300 and $500^{\circ} \mathrm{C}$ are field effect and chemical passivation, respectively. The field-effect passivation vanishes upon oxidation of the spin-on film, leading to the observed breakdown of surface passivation.

The PES measurements show that the formation of a$\mathrm{Si}: \mathrm{H}$ starts if the temperature is increased to about 350 and $400^{\circ} \mathrm{C}$. The passivation quality decreases again for these conversion temperatures. Masuda et al. have shown that the hydrogen density in the layer is strongly decreased, upon increasing the conversion temperature in this range and that the tetrahedral a-Si:H bond structure starts to form at $350^{\circ} \mathrm{C}^{25}$. Therefore $\tau_{15}$ is likely decreased because $\mathrm{Si}-\mathrm{H}$ bonds break, and weak $\mathrm{Si}-\mathrm{Si}$ and Si-dangling bonds are generated, leading to increased 


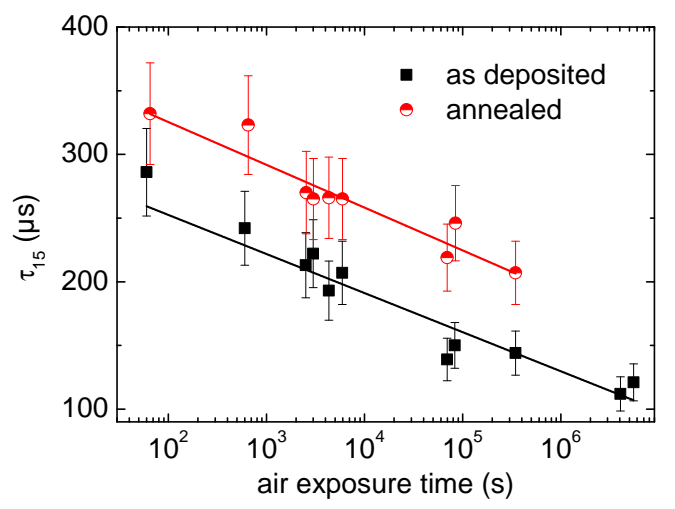

FIG. 4. Dependence of the minority charge carrier lifetime at an injection level of $10^{15} \mathrm{~cm}^{3}\left(\tau_{15}\right)$ for samples with $60 \mathrm{~nm}$ spin-coated a-Si:H on the air exposure time after film conversion. Values for as deposited and annealed $\left(2 \mathrm{~min}\right.$ at $300^{\circ} \mathrm{C}$ ) samples are displayed.

bulk defect density in the films and thus to an increased defect density at the interface to the crystalline silicon. Investigating the $\mathrm{Si}-\mathrm{H}$ bond distribution using infrared spectroscopy, or $\mathrm{H}$ outdiffusion could support this conclusions, but is difficult due to oxidation of the polysilane and the lack of in-situ measurement possibilities. Detailed accounts on changes in PECVD grown a-Si:H upon changes in the $\mathrm{H}$ microstructure can be found in the literature ${ }^{28}$. In this process the dangling bond concentration is influenced both by $\mathrm{Si}-\mathrm{H}$-bond breaking and hydrogen outdiffusion, as well as by $\mathrm{Si}$-Si-bond formation. The highest $\tau_{15}$ is reached at $500^{\circ} \mathrm{C}$, with $\approx 200 \mu \mathrm{s}$. Apart from a low defect density enabled by the passivation layer, it is also important, to obtain a long-term stable passivation. A-Si:H is known to degrade under illumination ${ }^{29}$. The defect density can be reduced again by thermal annealing at temperatures between 200 and $300^{\circ} \mathrm{C}$. Thus the degradation is reversible. Another reason for reversible degradation is found for liquid processed a-Si:H. $\tau_{15}$ of samples processed at 500 and $600^{\circ} \mathrm{C}$ degrades with increasing air exposure time of the samples. Furthermore it can be increased above its initial value using a thermal annealing step at $300^{\circ} \mathrm{C}$ for $2 \mathrm{~min}$. This degradation is reversible using air exposure and annealing cycles. Thus the stability of liquid processed a$\mathrm{Si}: \mathrm{H}$ is comparable to that of PECVD a-Si:H. The dependence of $\tau_{15}$ on air exposure time is shown in Fig. 4. The degradation of $\tau_{15}$ follows the same exponential decrease for as deposited and annealed samples. Therefore the reversible degradation mechanism is likely the same in both cases. Additionally, no degradation of $\tau_{15}$ is measured, if the samples are stored in nitrogen atmosphere and the degradation is the same for storage in the dark, or under daylight illumination. Therefore this degradation is either related to oxygen, or more likely water and furthermore not relevant for device applications, as passivation layers used in devices are encapsulated, or covered by other layers.

The best recoverable minority carrier lifetime reached with spin-coated a-Si:H, in the experiments presented up to this point, is only about $200 \mu \mathrm{s}$. A possible reason is that polysilane layers converted to a-Si:H are known to contain only a few atomic percent of hydrogen ${ }^{25}$, whereas a high atomic hydrogen content at the amorphous/crystalline interface is known to be a prerequisite for high quality interface passivation ${ }^{30}$. Thus it could be beneficial for the interface passivation, if the hydrogen content of the thermally converted layers and at the amorphous-crystalline interface is increased, e.g. using the diffusion of hydrogen from a hydrogen plasma through the a-Si:H layer to the a-Si:H/c-Si-interface ${ }^{15}$.

Therefore $90 \mathrm{~nm}$ thick liquid processed a-Si:H layers were annealed and afterwards exposed to a hydrogen plasma. The annealing step increases $\tau_{15}$ from $\approx 300 \mu$ s to $\approx$ $500 \mu \mathrm{s}$ and the subsequent hydrogen plasma treatment leads to a further increase to $\approx 1.37 \mathrm{~ms}$. This lifetime value corresponds to an excellent implied open circuit voltage $^{16}$ of $724 \mathrm{mV}$. This shows that very high quality passivation with liquid processable a- $\mathrm{Si}: \mathrm{H}$ layers is possible after hydrogen plasma treatment. The high thickness of these films precludes, for the moment, their use as buffer layers in a-Si:H/c-Si-solar cells.

In summary, using information from XPS the conversion temperature of neopentasilane to amorphous silicon was identified to be in the range of 300 to $350^{\circ} \mathrm{C}$. Furthermore it was shown that liquid neopentasilane precursors can be used to prepare high quality a-Si:H passivation layers for state of the art crystalline silicon surface passivation. Currently these a-Si:H layers are not suited for application in a-Si:H/c-Si solar cells due to their high valence band tail urbach energies of about 80 to $120 \mathrm{meV}$ and the high thicknesses needed for passivation.

To enable application of the investigated layers in a$\mathrm{Si}: \mathrm{H} / \mathrm{c}-\mathrm{Si}$ solar cells it will be crucial to improve their structural quality and increase their hydrogen content, resulting in an improved a-Si:H/c-Si interface and thinner passivation layers. Homogeneous deposition of a few nanometer thin layers with spin-coating is challenging, but spray coating has already been applied to deposit liquid silicon precursors ${ }^{31}$ for thin-film solar cells and could be used for the deposition of thin passivation layers. Moreover using spray coating could enhance the material quality and enable the usage of multi-chamber systems to conduct spray-coating and hydrogen plasma treatment, in the same system. Once these challenges are overcome, liquid processed a-Si:H offers great promise for development of solution processable a-Si:H layers for photovoltaic and other electronic applications and may present a first step towards solution processable, printable silicon based solar cell structures. 


\section{AKKNOWLEDGEMENTS}

The authors would like to thank Jasmin Lehmkuhl and Tobias Gergs for samples preparation, as well as Thomas Lußky for technical assistance.

${ }^{1}$ M. Tanaka, M. Taguchi, T. Matsuyama, T. Sawada, S. Tsuda, S. Nakano, H. Hanafusa, and Y. Kuwano, Jpn. J. Appl. Phys. 31, 3518 (1992).

${ }^{2}$ J. Bullock, A. Thomson, A. Cuevas, B. Veith, J. Schmidt, and A. Karkkainen, Phys. Status Solidi RRL. 7, 530 (2013).

${ }^{3}$ C.-M. Yang, D.-S. Kim, S.-G. Lee, J.-H. Lee, Y. S. Lee, and J.-H. Lee, IEEE Electron Device Lett. 33, 564 (2012).

${ }^{4}$ D. M. Garner and G. A. Amaratunga, Solid-State Electronics 43, 1973 (1999).

${ }^{5}$ M. Taguchi, A. Yano, S. Tohoda, K. Matsuyama, Y. Nakamura, T. Nishiwaki, K. Fujita, and E. Maruyama, IEEE J. Photovolt. 4, 96 (2014).

${ }^{6}$ T. Shimoda, Y. Matsuki, M. Furusawa, T. Aoki, I. Yudasaka, H. Tanaka, H. Iwasawa, D. Wang, M. Miyasaka, and Y. Takeuchi, Nature 440, 783 (2006).

${ }^{7}$ T. Sontheimer, D. Amkreutz, K. Schulz, P. H. Wöbkenberg, C. Guenther, V. Bakumov, J. Erz, C. Mader, S. Traut, F. Ruske, et al., Adv. Mat. Interfaces 1, 1300046 (2014).

${ }^{8}$ J. D. Holmes, K. P. Johnston, R. C. Doty, and B. A. Korgel, Science 287, 1471 (2000).

${ }^{9}$ T. Bronger, P. H. Wöbkenberg, J. Wördenweber, S. Muthmann, U. W. Paetzold, V. Smirnov, S. Traut, U. Dagkaldiran, S. Wieber, M. Cölle, et al., Adv. Energy Mater. 4, 1301871 (2014).

${ }^{10}$ T. Masuda, N. Sotani, H. Hamada, Y. Matsuki, and T. Shimoda, Appl. Phys. Lett. 100, 253908 (2012).

${ }^{11}$ M. Fujiki, Y. Kawamoto, M. Kato, Y. Fujimoto, T. Saito, S.-i. Hososhima, and G. Kwak, Chem. Mater. 21, 2459 (2009).

${ }^{12}$ T. D. Bogart, D. Oka, X. Lu, M. Gu, C. Wang, and B. A. Korgel, ACS Nano 8, 915 (2014).
${ }^{13}$ B. Veith, F. Werner, D. Zielke, R. Brendel, and J. Schmidt, Energy Procedia 8, 307 (2011).

${ }^{14}$ K. H. Chung, J. C. Sturm, E. Sanchez, K. K. Singh, and S. Kuppurao, Semicond. Sci. Technol. 22, S158 (2007).

${ }^{15}$ M. Mews, T. F. Schulze, N. Mingirulli, and L. Korte, Appl. Phys. Lett. 102, 122106 (2013).

${ }^{16}$ R. A. Sinton and A. Cuevas, Appl. Phys. Lett. 69, 2510 (1996).

${ }^{17}$ M. Sebastiani, L. Di Gaspare, G. Capellini, C. Bittencourt, and F. Evangelisti, Phys. Rev. Lett. 75, 3352 (1995).

${ }^{18}$ L. Korte and M. Schmidt, J. Non-Cryst. Solids 354, 2138 (2008).

${ }^{19}$ G. Hollinger, Y. Jugnet, P. Pertosa, and T. M. Duc, Chem. Phys. Lett. 36, 441 (1975).

${ }^{20}$ G. Hollinger and F. J. Himpsel, Phys. Rev. B 28, 3651 (1983).

${ }^{21}$ H. Nakano, M. Nakano, K. Nakanishi, D. Tanaka, Y. Sugiyama, T. Ikuno, H. Okamoto, and T. Ohta, J. Am. Chem. Soc. 134, 5452 (2012).

${ }^{22}$ W. Hansch, A. Nakajima, and S. Yokoyama, Appl. Phys. Lett. 75, 1535 (1999).

${ }^{23}$ T. F. Schulze, F. Ruske, B. Rech, and L. Korte, Phys. Rev. B 83, 165314 (2011).

${ }^{24}$ M. J. Powell and S. C. Deane, Phys. Rev. B 48, 10815 (1993).

${ }^{25}$ T. Masuda, Y. Matsuki, and T. Shimoda, Thin Solid Films 520, 6603 (2012).

${ }^{26}$ G. Dingemans and W. Kessels, J. Vac. Sci. Technol. A 30, 040802 (2012).

${ }^{27}$ C. Leendertz, N. Mingirulli, T. F. Schulze, J.-P. Kleider, B. Rech, and L. Korte, Appl. Phys. Lett. 98, 202108 (2011).

${ }^{28}$ S. Lebib and P. Roca i Cabarrocas, J. Appl. Phys. 97, 104334 (2005).

${ }^{29}$ H. Plagwitz, B. Terheiden, and R. Brendel, J. Appl. Phys. 103, 094506 (2008).

${ }^{30}$ H. Fujiwara and M. Kondo, Appl. Phys. Lett. 86, 032112 (2005).

${ }^{31} \mathrm{~T}$. Shimoda and T. Masuda, in MRS Spring Meeting (2014). 